\title{
Dimensional Reduction Study of Piezoelectric Ceramics Constitutive Equations from 3-D to 2-D and 1-D
}

\author{
Meiling Zhu and Glenn Leighton
}

\begin{abstract}
Accurate performance evaluation is crucial to the design and development of macro/micro-sized piezoelectric devices, and key to this is the proper use of the stiffness/ compliance and piezoelectric coefficients of the piezoelectric ceramics involved. Although the literature points out effective piezoelectric coefficients $e_{31, f}$ and $d_{33, f}$ for thin film materials and reduced dimensionality of equations for bulk material, the elastic and piezoelectric coefficients remain unchanged from the 3-D equations in most reported 1-D and 2-D analyses of the macro/micro-sized devices involving the $e$ form of the constitutive equations. The use of unchanged coefficients leads to variations between numerically predicted and experimental results in most devices. To understand effects of the dimensional reduction from 3-D to 2-D and 1-D on stiffness/compliance and piezoelectric coefficients, this paper derives the 2-D and 1-D constitutive equations from the $3-D$ equations, focusing on the discussion of often-required device configurations for sensor and actuator design and analysis. Two modified coefficients are proposed, termed reduced and enhanced, which enable better understanding of effects of the dimensional reduction and also effects on the design and analysis of sensors and actuators.
\end{abstract}

\section{INTRODUCTION}

$\mathrm{P}$ iezoelectric materials are finding increased applications in a variety of macro/micro-sized sensors and actuators through the direct and/or converse piezoelectric effects. A large number of these devices utilize either the longitudinal piezoelectric coefficient-for example, thin film bulk acoustic wave resonators (TBAR) and filters [1]-[3], or transverse piezoelectric coefficient - for example, unimorph/bimorph structures such as cantilevers, fixedfixed beams, and membranes [4-6]. Accurate evaluation of macro/micro-sized piezoelectric device performance is crucial to design and development, requiring proper use of the stiffness/compliance and piezoelectric coefficients involved because piezoelectric device performance such as static and dynamic responses are determined by these coefficients. The literature points out effective piezoelectric coefficients $e_{31, f}$ and $d_{33, f}$ for thin film materials [7-9] and reduced dimensionality equations for bulk materials [10], [11], but there is still a lack of understanding of piezoelectric ceramics constitutive equations in the dimensional reduction. This is due to the fact that the following $e$ or $d$

Manuscript received March 10, 2008; accepted May 30, 2008. This work received support from EPSRC Platform (EP/D506638/1).

The authors are with the Microsystems and Nanotechnology Centre, School of Applied Sciences, Cranfield University, Cranfield, Bedfordshire MK43 0AL, UK (e-mail:m.zhu@cranfield.ac.uk)

Digital Object Identifier 10.1109/TUFFC.945 forms of the constitutive equations for $z$-polarized piezoelectric ceramics are used for 2-D analyses of macro/microsized devices. In most of the literature and textbooks, the stiffness, compliance, and piezoelectric coefficients remain unchanged from the $3-\mathrm{D}$ constitutive equations:

$$
\begin{aligned}
& {\left[\begin{array}{c}
\sigma_{x} \\
\sigma_{y} \\
\sigma_{x y}
\end{array}\right]=\left[\begin{array}{ccc}
c_{11} & c_{12} & 0 \\
c_{12} & c_{22} & 0 \\
0 & 0 & c_{66}
\end{array}\right]\left\{\begin{array}{c}
\varepsilon_{x} \\
\varepsilon_{y} \\
\varepsilon_{x y}
\end{array}\right\}-\left[\begin{array}{lll}
e_{31} & e_{31} & 0
\end{array}\right]^{\mathrm{T}} E_{3}} \\
& D_{3}=\left[\begin{array}{lll}
e_{31} & e_{31} & 0
\end{array}\right]\left\{\begin{array}{c}
\varepsilon_{x} \\
\varepsilon_{y} \\
\varepsilon_{x y}
\end{array}\right\}+\varepsilon_{3}^{\varepsilon} E_{3} \\
& \left\{\begin{array}{c}
\varepsilon_{x} \\
\varepsilon_{y} \\
\varepsilon_{x y}
\end{array}\right\}=\left[\begin{array}{ccc}
s_{11} & s_{12} & 0 \\
s_{12} & s_{22} & 0 \\
0 & 0 & s_{66}
\end{array}\right]\left[\begin{array}{c}
\sigma_{x} \\
\sigma_{y} \\
\sigma_{x y}
\end{array}\right]+\left[\begin{array}{lll}
d_{31} & d_{31} & 0
\end{array}\right]^{\mathrm{T}} E_{3} \\
& D_{3}=\left[\begin{array}{lll}
d_{31} & d_{31} & 0
\end{array}\right]\left[\begin{array}{c}
\sigma_{x} \\
\sigma_{y} \\
\sigma_{x y}
\end{array}\right]+\varepsilon_{3}^{\sigma} E_{3}
\end{aligned}
$$

In these equations $\sigma_{i}, \varepsilon_{i}, c_{i j}$, and $s_{i j}$ denote in turn components of the stress and strain vectors and components of stiffness and compliance matrices measured at constant electrical fields. $E_{3}$ and $D_{3}$ are the $z$-components of the electric field and displacement, $e_{i j}$ and $d_{i j}$ are the piezoelectric stress and strain coefficients measured at constant strains and constant stresses, and $\varepsilon_{3}^{\varepsilon}$ and $\varepsilon_{3}^{\sigma}$ are the $z$-components of dielectric coefficients measured at constant strains and at constant stresses. From (1)-(4) it appears that $e_{31}$ (or $d_{31}$ ) alone when used for 2-D structural analyses - that is, $e_{33}$ (or $d_{33}$ ) - has no effect. We found that this is true for (3) and (4) but not generally for (1) and (2).

The main motivation for this paper is to 1) understand effects of the dimensional reduction from 3-D to 2-D and 1-D on stiffness/compliance and piezoelectric coefficients, which are often required in piezoelectric sensor and actuator design and analysis; and 2) to make them available to piezoelectric researchers and users at a level that enables them to accurately evaluate piezoelectric devices' performance. This paper will therefore use the $3-\mathrm{D}$ constitutive equations to systematically derive $2-\mathrm{D}$ and $1-\mathrm{D}$ constitutive equations for piezoelectric ceramic materials by considering the following specific cases which feature modified elastic, piezoelectric, and dielectric constants: 1) 2-D plane stress state, 2) 1-D beam with $x$-directed stress, 3) 1-D bar with $z$-directed stress, and 4) thin/thick film clamped on a 
substrate with $z$-directed strain. Two modified coefficients are presented in this paper, termed reduced and enhanced, to enable better understanding of the effects of the dimensional reduction on piezoelectric material coefficients and their impact on the design and analysis of sensors and actuators for diverse readers with interests ranging from piezoelectric bulk and thin film materials to piezoelectric devices. The importance of these modified coefficients to the design and analysis of sensors and actuators has also been demonstrated by using their calculated values to determine the tip displacement of a micro-cantilever and the charge output of a triaxial accelerometer.

\section{Derivations of 1-D And 2-D Constitutive EQUATIONS}

All the derivations of the $e$ or $d$ forms of the constitutive equations in the 1-D and 2-D analyses below start from the 3-D constitutive equations and in all the cases studied the electric field is applied in the $z$-direction (thickness) only while the piezoelectric materials are $c$-axis oriented or ferroelectric poled in the $z$-direction. In addition, the polarized piezoelectric ceramics are transversely isotropic in the $x-y$ plane. Their linear behavior described by (5) and (6) is the same as crystals having $6 \mathrm{~mm}$ symmetry.

\section{III. $E$ Form of Constitutive Equations}

The 3-D e form of the constitutive equations is

$$
\begin{aligned}
&\left\{\begin{array}{c}
\sigma_{x} \\
\sigma_{y} \\
\sigma_{z} \\
\sigma_{y z} \\
\sigma_{x z} \\
\sigma_{x y}
\end{array}\right\}= {\left[\begin{array}{llllll}
c_{11} & c_{12} & c_{13} & & \\
c_{12} & c_{11} & c_{13} & & \\
c_{13} & c_{13} & c_{33} & & \\
& & & c_{44} & & \\
& & & & c_{44} & \\
\left\{\begin{array}{c}
D_{1} \\
D_{2} \\
D_{3}
\end{array}\right\}= & {\left[\begin{array}{cccccc}
0 & 0 & 0 & 0 & e_{15} & 0 \\
0 & 0 & 0 & e_{15} & 0 & 0 \\
e_{31} & e_{31} & e_{33} & 0 & 0 & 0
\end{array}\right]\left[\begin{array}{l}
\varepsilon_{x} \\
\varepsilon_{y} \\
\varepsilon_{z} \\
\varepsilon_{y z} \\
\varepsilon_{x z} \\
\varepsilon_{x y}
\end{array}\right\}\left\{\begin{array}{l}
E_{1} \\
E_{2} \\
E_{3}
\end{array}\right\}} \\
e_{31} & e_{31} & e_{33} & 0 & 0 & 0
\end{array}\right] } \\
&+\left[\begin{array}{cccc}
\varepsilon_{11} & 0 & 0 \\
0 & \varepsilon_{11} & 0 \\
0 & 0 & \varepsilon_{33}
\end{array}\right]\left\{\begin{array}{l}
e_{24} \\
E_{1} \\
E_{2} \\
E_{3}
\end{array}\right\} .
\end{aligned}
$$

\section{A. Plane Stress State}

In practical engineering problems one is often interested in a state of plane stress, as this stress state is often re-

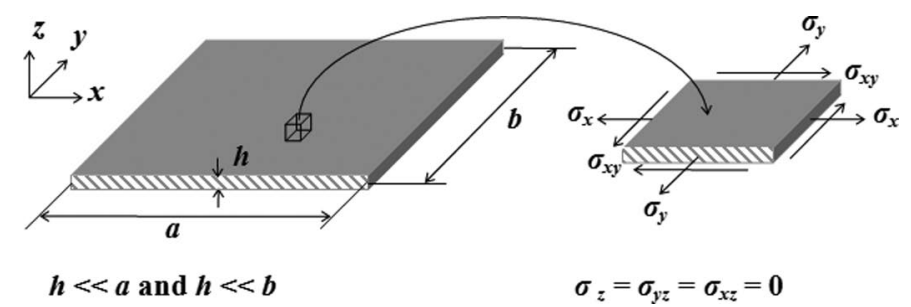

Fig. 1. Plane stress state.

quired to design macro/micro-sized plane structures such as diaphragms or membranes for pumps and microphones [12]. In the case shown in Fig. 1, the thickness $h$ of the plate is substantially less than the in-plane ( $x-y$ plane) dimensions and so

$$
\sigma_{z}=0, \quad \sigma_{y z}=0, \quad \sigma_{x z}=0 .
$$

In the case studied here the voltage is only applied in the $z$-direction, and so we have

$$
E_{1}=E_{2}=D_{1}=D_{2}=0 \text { and } \quad E_{3} \neq 0, \quad D_{3} \neq 0
$$

Substituting (7) and (8) into the term $\sigma_{z}$ in (5) gives

$$
\varepsilon_{z}=-\frac{c_{13}}{c_{33}} \varepsilon_{x}-\frac{c_{13}}{c_{33}} \varepsilon_{y}+\frac{e_{33}}{c_{33}} E_{3}
$$

Then substituting (9) into the terms $\sigma_{x}$ and $\sigma_{y}$ in (5) and the term $D_{3}$ into (6), we obtain

$$
\begin{aligned}
& {\left[\begin{array}{c}
\sigma_{x} \\
\sigma_{y} \\
\sigma_{x y}
\end{array}\right]=\left[\begin{array}{ccc}
c_{11,2-\mathrm{D}} & c_{12,2-\mathrm{D}} & 0 \\
c_{12,2-\mathrm{D}} & c_{11,2-\mathrm{D}} & 0 \\
0 & 0 & c_{66}
\end{array}\right]\left\{\begin{array}{c}
\varepsilon_{x} \\
\varepsilon_{y} \\
\varepsilon_{x y}
\end{array}\right\}-\left[\begin{array}{lll}
e_{31,2-\mathrm{D}} & e_{31,2-\mathrm{D}} & 0
\end{array}\right]^{\mathrm{T}} E_{3}} \\
& D_{3}=\left[\begin{array}{lll}
e_{31,2-\mathrm{D}} & e_{31,2-\mathrm{D}} & 0
\end{array}\right]\left\{\begin{array}{c}
\varepsilon_{x} \\
\varepsilon_{y} \\
\varepsilon_{x y}
\end{array}\right\}+\varepsilon_{3,2-\mathrm{D}}^{\varepsilon} E_{3}
\end{aligned}
$$

where

$$
\begin{aligned}
& c_{11,2-\mathrm{D}}=c_{11}-\frac{c_{13}^{2}}{c_{33}}, \quad c_{12,2-\mathrm{D}}=c_{12}-\frac{c_{13}^{2}}{c_{33}} \\
& e_{31,2-\mathrm{D}}=e_{31}-e_{33} \frac{c_{13}}{c_{33}}, \quad \varepsilon_{3,2-\mathrm{D}}^{\varepsilon}=\varepsilon_{3}^{\varepsilon}+\frac{e_{33}^{2}}{c_{33}} .
\end{aligned}
$$

It can be seen from (12) that the 2-D stiffness, piezoelectric, and dielectric coefficients- $c_{i j, 2-\mathrm{D}}, e_{31,2-\mathrm{D}}$, and $\varepsilon_{3,2-\mathrm{D}}^{\varepsilon}$, respectively -introduced in (10) and (11) are either greater or smaller than their 3-D counterparts. Where smaller $\left(c_{i j, 2-\mathrm{D}}\right)$ they are given the term reduced and where greater $\left(e_{31,2-\mathrm{D}}\right.$ and $\left.\varepsilon_{3,2-\mathrm{D}}^{\varepsilon}\right)$ they are termed enhanced. The enhanced designation for $e_{31,2-\mathrm{D}}$ might not be imme- 


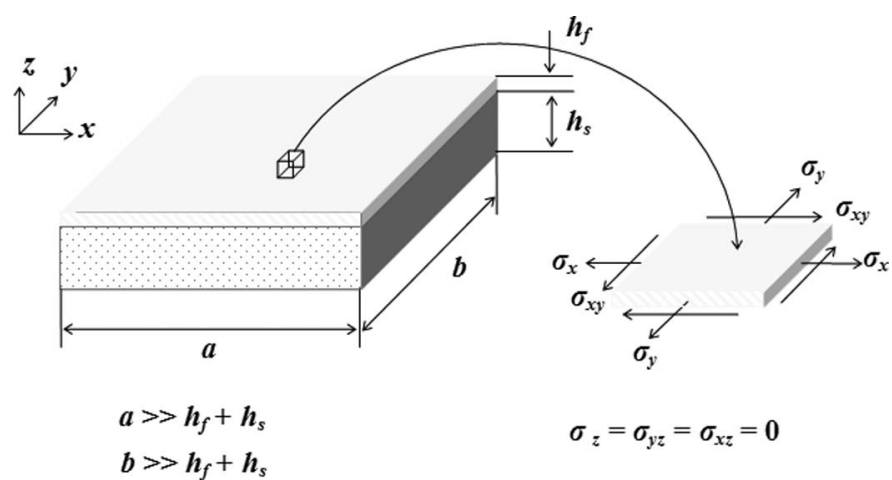

Fig. 2. Thin/thick film $e_{31, f}$ derived from plane stress state.

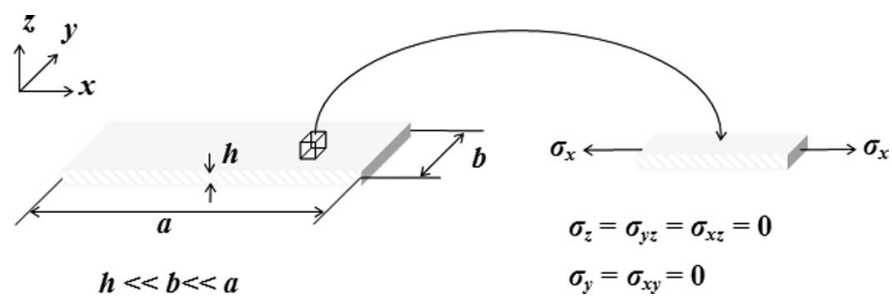

Fig. 3. 1-D beam with the $x$-directional stress.

diately apparent but is due to the differing signs of $e_{31}$ and $e_{33}$, which agrees with the conclusions of [10] and [11].

Although the enhanced piezoelectric coefficient $e_{31,2-\mathrm{D}}$ was derived for the plane stress state, it can be found to be exactly the same as the effective piezoelectric coefficient $e_{31, f}$, which is a practical measurement of $e_{31}$ for a piezoelectric thin/thick film on a substrate [7-9]. So it can be said that $e_{31, f}$ would also be measured in the plane stress state, shown in Fig. 2 where the thickness of piezoelectric $h_{f}$ is comparable to that of the structure $h_{s}$, but it should be borne in mind that this is only true if the dimensions of the electrode area of the sample are significantly larger than the sample thickness $\left(h_{s}+h_{p}\right)$ to ensure the condition for the plane stress state.

Apart from the already mentioned $e_{31, f}$, reduced and enhanced coefficients are rarely used in 2-D macro/microsized structural or device design and analyses when the $e$ form of the constitutive equations is used, which would lead to the calculated charge being lower than that generated in an actual device, whereas for actuator analyses, the calculated deformation is lower than that of an actual device. So the analyses of macro/micro -sized piezoelectric device performance would be significantly underestimated if (1) and (2) were used.

\section{B. 1-D Beam With the $x$-Directed Stress}

In this case, depicted in Fig. 3, the $x$ dimension is significantly larger than the $y$ and $z$ dimensions and so we have a 1-D beam. Such a beam structure can be used to design cantilevers for RF-MEMS switches, resonators, and filters, as well as pressure and accelerometer sensors. Because the thickness is taken as small and all the stresses are zero on the $2 x$ - $z$ and $y$ - $z$ surfaces, these stresses cannot differ appreciably from zero in the interior and give

$$
\sigma_{z}=\sigma_{y z}=\sigma_{x z}=0 .
$$

Similarly, because the width is considered very small, the stresses are

$$
\sigma_{y}=\sigma_{x y}=\sigma_{y z}=0
$$

It should be known that (13) and (14) apply throughout the volume of the beam.

For the length, the only finite dimension, the stresses on the surface, $\sigma_{x z}$ and $\sigma_{x y}$, have already been found to be zero so that the only stress different from zero in the interior is $\sigma_{x}$.

Substituting (13) and (14) into (5)

$$
\left|\begin{array}{cc}
c_{11} & c_{13} \\
c_{13} & c_{33}
\end{array}\right|\left\{\begin{array}{l}
\varepsilon_{y} \\
\varepsilon_{z}
\end{array}\right\}=\left\{\begin{array}{l}
e_{31} E_{3}-c_{12} \varepsilon_{x} \\
e_{33} E_{3}-c_{12} \varepsilon_{x}
\end{array}\right\}
$$

and then solving for $\varepsilon_{y}$ and $\varepsilon_{z}$ in terms of $\varepsilon_{x}$ and $E_{3}$ gives

$$
\left\{\begin{array}{l}
\varepsilon_{y} \\
\varepsilon_{z}
\end{array}\right\}=\frac{1}{c_{11} c_{33}-c_{13}^{2}}\left|\begin{array}{cc}
c_{33} & -c_{13} \mid \\
-c_{13} & c_{11}
\end{array}\right|\left\{\begin{array}{l}
e_{31} E_{3}-c_{12} \varepsilon_{x} \\
e_{33} E_{3}-c_{12} \varepsilon_{x}
\end{array}\right\} \text {. }
$$

Substituting these solutions into the terms $\sigma_{x}$ and $D_{3}$ gives

$$
\begin{aligned}
& \sigma_{x}=c_{11, b} \varepsilon_{x}-e_{31, b} E_{3} \\
& D_{3}=e_{31, b} \varepsilon_{x}+\varepsilon_{3, b}^{\varepsilon} E_{3}
\end{aligned}
$$

where

$$
\begin{aligned}
c_{11, b} & =c_{11}-\frac{c_{12}^{2} c_{33}-2 c_{12} c_{13}^{2}+c_{11} c_{13}^{2}}{c_{11} c_{33}-c_{13}^{2}} \\
e_{31, b} & =e_{31}\left(1-\frac{c_{12} c_{33}-c_{13}^{2}}{c_{11} c_{33}-c_{13}^{2}}\right)-e_{33} \frac{c_{13}\left(c_{11}-c_{12}\right)}{c_{11} c_{33}-c_{13}^{2}} \\
\varepsilon_{3, b}^{\varepsilon} & =\varepsilon_{3}^{\varepsilon}+e_{31} \frac{e_{31}\left(c_{33}-c_{13}\right)+e_{33}\left(c_{11}-c_{13}\right)}{c_{11} c_{33}-c_{13}^{2}} .
\end{aligned}
$$

As can be seen in the preceding equations, the coefficients are reduced or enhanced as mentioned in Section III.A, and similar conclusions about their effects can be drawn as given previously with reference to (10) and (11).

\section{1-D Bar With the z-Directed Stress}

In this case, depicted in Fig. 4, the dimension in the $z$ direction is significantly larger than the in-plane dimensions ( $x$ - $y$ plane) and so all the stresses are zero on the two surfaces normal to $x$ and $y$, which gives 


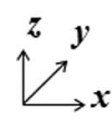

$$
\sigma_{x}=\sigma_{y}=\sigma_{x y}=0 \quad \text { and } \quad \sigma_{z} \neq 0
$$

Substituting (19) into the $e$ form of the constitutive equations (5)-(6) and then using similar notation to (17) gives

$$
\begin{aligned}
\sigma_{z} & =c_{33, b} \varepsilon_{z}-e_{33, b} E_{3} \\
D_{3} & =e_{33, b} \varepsilon_{z}+\varepsilon_{3, b}^{\varepsilon} E_{3}
\end{aligned}
$$

where

$$
\begin{aligned}
c_{33, b} & =c_{33}-\frac{2 c_{13}^{2}}{c_{11}+c_{12}} \\
\mathrm{e}_{33, b} & =e_{33}-\frac{2 c_{13}}{c_{11}+c_{12}} e_{31} \\
\varepsilon_{3, b}^{\varepsilon} & =\varepsilon_{3}^{\varepsilon}+\frac{2 e_{31}^{2}}{c_{11}+c_{12}} .
\end{aligned}
$$

Reduced and enhanced coefficients result, with effects as noted previously in the discussion of (10) and (11).

\section{D Form of the Constitutive Equations}

\section{A. Plane Stress State}

Using a derivation similar to that leading to (10) and (11) but starting from the 3-D constitutive equations in the $d$ form, it can be shown that (3) and (4) are the correct forms for the 2-D piezo-plane stress state. Therefore, in this case the $d$ form coefficients that appear in the 3-D constitutive equations are used without alteration, which means that only $d_{31}$ is needed for 2-D plate structural analyses whereas for the $e$ form of the constitutive equations both $e_{31}$ and $e_{33}$ need to be included.

\section{B. 1-D Beam or Bar With $x$ - and $z$-Directed Stress, Respectively}

Similar conclusions as in the preceding discussion of the $d$ form of the constitutive equations for the 2-D plane

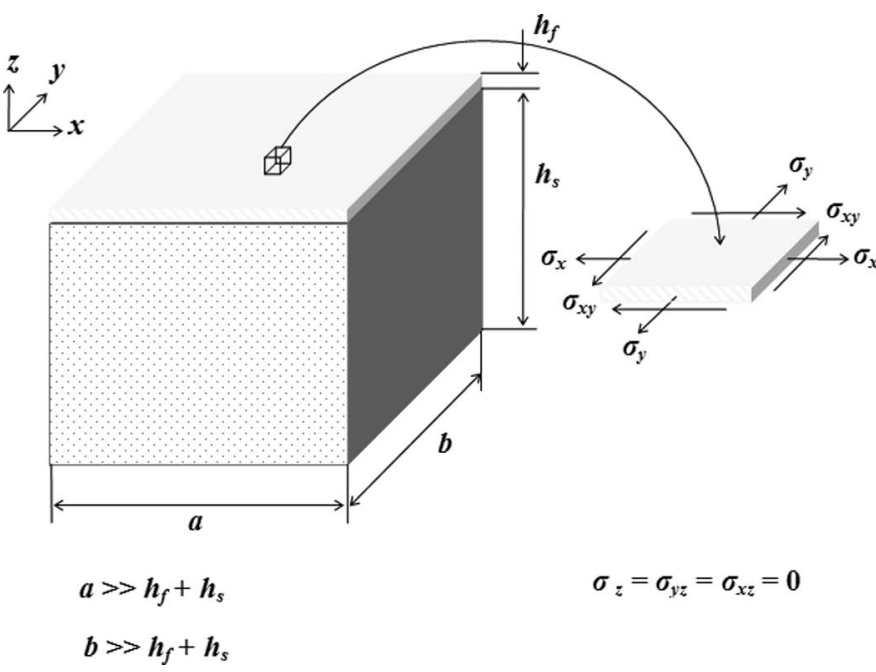

Fig. 5. Thin/thick film clamped on substrate with the $z$-directional strain.

stress state can be reached for the case discussed in Section III.B and III.C. That is, when the $d$ form of the constitutive equations are used in 1-D beam analyses for sensors and actuators, the coefficients are simply those from the 3 -D constitutive equations without alteration.

\section{Thin/Thick Film Clamped on a Substrate With THE $Z$-DireCted Strain}

The thin/thick film clamped on a substrate depicted in Fig. 5, with the $z$-directed strain and the thickness of piezoelectric film $h_{f}$ significantly less than the thickness of the substrate $h_{s}$, is the exceptional case of the $e$ form of the constitutive equations in which the coefficients remain unchanged from the 3 -D values whereas the $d$ form coefficients are changed. The reasons for this exception are given through the following derivation.

Since the thin/thick film is clamped to the substrate, the strains, $\varepsilon_{x}$ and $\varepsilon_{y}$, cannot differ appreciably from zero in the substrate, and so

$$
\varepsilon_{x}=\varepsilon_{y}=0
$$

Substituting (22) into the $e$ form of the constitutive equation (5) gives

$$
\begin{gathered}
\sigma_{z}=c_{33} \varepsilon_{z}-e_{33} E_{3} \\
D_{3}=e_{33} \varepsilon_{z}+\varepsilon_{3}^{\varepsilon} E_{3}
\end{gathered}
$$

and it can be seen that the coefficients are the same as given in (5) and (6) for the full 3-D treatment. The TBAR is an example of a device composed of a thin film clamped on a substrate and accordingly the analysis, generally 1-D, of the $z$-directed longitudinal vibration it produces during operation utilizes $e_{33}$ rather than $d_{33}$. However, one needs to consider that the condition $\varepsilon_{x}=\varepsilon_{y}=0$ can only be met 
if the substrate is not subjected to a bending deformation, and this can only be the case if the strain is purely in the $z$-direction, giving a "piston mode" vibration.

In contrast the $d$ form of the constitutive equations changes to

$$
\begin{aligned}
\varepsilon_{z} & =s_{33, f} \sigma_{z}+d_{33, f} E_{3} \\
D_{3} & =d_{33, f} \sigma_{z}+\varepsilon_{33, f}^{\sigma} E_{3}
\end{aligned}
$$

where

$$
\begin{aligned}
s_{33, f} & =-\frac{2 s_{13}^{2}}{s_{11}+s_{12}}+s_{33}, \\
d_{33, f} & =-\frac{2 s_{13}}{s_{11}+s_{12}} d_{31}+d_{33}, \\
\varepsilon_{33, f}^{\sigma} & =\varepsilon_{33}^{\sigma}-\frac{2 d_{31}^{2}}{s_{11}+s_{12}}
\end{aligned}
$$

The minus sign in the $-2 s_{13}^{2} /\left(s_{11}+s_{12}\right)$ term combined with the fact that $s_{13}$ and $d_{31}$ are always negative whereas $d_{33}$ is always positive means that $s_{33, f}$ and $d_{33, f}$ are smaller than $s_{33}$ and $d_{33}$, respectively, which is the opposite of what was found earlier for $e_{33, b}$ and $c_{33, b}$. Therefore, in this case, the compliance and piezoelectric coefficients are reduced whereas the stiffness is increased.

Here it is worthwhile mentioning that when using this technique to measure piezoelectric thin film coefficients, $d_{33, f}$ is obtained rather than $d_{33}$. It also should be ensured that the electrode in-plane dimensions are much smaller than the substrate thickness; otherwise, there will be a significant unwanted contribution from $e_{31, f}$.

\section{VI. $D_{31}$ Relation to $E_{31}$ AND $E_{33}$}

Having derived the $e$ and $d$ forms of the 1-D and 2-D constitutive equations for the common cases, the relationship between $d$ and $e$ is now examined to further understand effects of the dimensional reduction by bringing the $e$ and $d$ forms of the constitutive equations together. Because the case in Section III-A has a slightly different relation, here we use the plane stress state for further understanding of this case. The derivation of relationships can start from substituting (10) into (3) and obtaining,

$$
[d]^{\mathrm{T}}=[s]\left[e_{2 D}\right]^{\mathrm{T}}
$$

Expanding (27) gives

$$
\frac{d_{31}}{s_{11}+s_{12}}=e_{31,2 D}=e_{31}-e_{33} \frac{c_{13}}{c_{33}} .
$$

According to [13], we have

$$
s_{11}=\frac{1}{E_{11}}, s_{12}=\frac{-\nu_{x y}}{E_{11}}, s_{13}=\frac{-\nu_{x z}}{E_{11}}
$$

$$
\begin{array}{r}
c_{13}=\left(\nu_{x z}+\nu_{x y} \nu_{x z}\right) \frac{E_{33}}{V}, \quad c_{33}=\left(1-\nu^{2}{ }_{x y}\right) \frac{E_{33}}{V} \\
V=\left[1-\nu_{x y}\left(\nu_{x y}+2 \nu^{2}{ }_{x z} \frac{E_{33}}{E_{11}}\right)-2 \nu^{2}{ }_{x z} \frac{E_{33}}{E_{11}}\right]
\end{array}
$$

where $E_{i i}$ is the Young's modulus in the $i$ direction; $v_{i j}$ are the Poisson's ratios as determined from the contraction in the $j$ direction caused by a tensile stress in the $i$ direction, and $V$ is related to the material Young's modulus and Poisson's ratios.

Therefore,

$$
\begin{gathered}
-\frac{s_{13}}{s_{11}+s_{12}}=\frac{\nu_{x z}}{1-\nu_{x y}} \\
\frac{c_{13}}{c_{33}}=\frac{v_{x z}}{1-v_{x y}}
\end{gathered}
$$

which yields

$$
\frac{c_{13}}{c_{33}}=-\frac{s_{13}}{s_{11}+s_{12}} .
$$

Substituting this result into (28) we have

$$
\frac{d_{31}}{s_{11}+s_{12}}=e_{31,2-\mathrm{D}}=e_{31}+e_{33} \frac{s_{13}}{s_{11}+s_{12}}
$$

giving

$$
d_{31}=e_{31}\left(s_{11}+s_{12}\right)+e_{33} s_{13} .
$$

Eq. (36) shows that $d_{31}$ can be represented by $e_{31}$ and $e_{33}$ through compliance coefficients, or in other words, for the plane stress state, $d_{31}$ alone controls plate deformations, but if $e$ coefficients are used, both $e_{31}$ and $e_{33}$ need to be included, as Muralt et al. [14] concur.

\section{Effect of Modified Coefficient on the Analysis of SEnsors and ACtuators}

To quantitatively evaluate the effects of using the reduced and enhanced coefficients in the constitutive equations in the analysis of sensor and actuator performance, the modified stiffness, piezoelectric, and dielectric coefficients for the plane stress state were calculated, as listed in Table I alongside the original coefficients. Both sets of coefficients were then used as parameters in 2 finite element modeling programs written using commercial software from ANSYS (ANSYS, Inc., Canonsburg, PA). The programs calculated the performance of 2 microdevices with integrated piezoelectric thin films: 1) a silicon cantilever with piezoelectric thin film actuation measuring $1000 \mu \mathrm{m} \times 250 \mu \mathrm{m} \times 15 \mu \mathrm{m}$, and 2) a triaxial microac- 
TABle I. Modified Material Coefficients Alongside the Original Coefficients.

\begin{tabular}{lcc}
\hline & Original & Modified \\
\hline$e_{31}\left(\mathrm{~cm}^{-2}\right)$ & -10.84 & -20.84 \\
$e_{33}\left(\mathrm{~cm}^{-2}\right)$ & 13.68 & - \\
$c_{11}(\mathrm{GPa})$ & 148 & 69.6 \\
$c_{12}(\mathrm{GPa})$ & 98.1 & 19.6 \\
$e_{33}$ & 759 & 903 \\
\hline
\end{tabular}

Note the lack of applicable values for $e_{33}$.

TABle II. Simulation Results for a Cantilever Actuator and AcCelerometer Using the Original and Modified Coefficients.

\begin{tabular}{lcc}
\hline & Original & Modified \\
\hline $\begin{array}{l}\text { Tip displacement of a cantilever when } 1 \mathrm{~V} \\
\text { voltage is applied to piezoelectric thin film }\end{array}$ & $2.13 \mu \mathrm{m}$ & $9.46 \mu \mathrm{m}$ \\
$\begin{array}{l}\text { Charge output of accelerometer under the } \\
\text { action of } 1 \mathrm{~g} \text { acceleration }\end{array}$ & $7.84 \mathrm{pC}$ & $20 \mathrm{pC}$ \\
\hline
\end{tabular}

celerometer with piezoelectric thin film sensing. The resulting tip displacement of the cantilever for the case of 1- $\mu \mathrm{m}$-thick piezoelectric thin film with $1 \mathrm{~V}$ applied voltage and the charge output of the accelerometer under action of $1 \mathrm{~g}$ acceleration were calculated and are listed in Table II for both the original and modified coefficients. The material parameters and geometrical dimensions for the simulations of the cantilever and accelerometer were taken from [15, Table 1] and from [16, Tables 2 and 3], respectively. It can be seen from Table II that the simulated tip displacement and charge based on analysis using the reduced and enhanced coefficients are different from the values calculated using the original coefficients and that the new coefficients can lead to accurate prediction of device performance, such as the static and dynamic responses of devices.

\section{Conclusions}

Accurate evaluation of macro/micro-sized piezoelectric device performance is dependent on the proper use of the relevant piezoelectric coefficients, which are therefore crucial for the design, analysis, and development of such devices. Although there are, as the literature has already pointed out, effective piezoelectric coefficients $e_{31, f}$ and $d_{33, f}$ for thin film materials, the elastic and piezoelectric coefficients still remain unchanged from the $3-\mathrm{D}$ equations in most of the reported macro/micro-sized leveled devices' 1-D and 2-D analyses involving the $e$ form of the constitutive equations. Two modified coefficients, termed reduced and enhanced, have been systematically introduced into the constitutive equations, enabling better understand- ing of the dimensional reduction from $3-\mathrm{D}$ to $2-\mathrm{D}$ and 1-D, and of how they affect design and analysis of sensors and actuators. In addition, this work is based on the 3-D constitutive equations and so is applicable to the whole range of piezoelectric ceramics from bulk down to thick/ thin films.

\section{ACKNOWLEDGEMENTS}

The authors would like to acknowledge support from EPSRC Platform (EP/D506638/1) and also would like to thank Mr. Robert Wright for helpful discussions.

\section{REFERENCES}

[1] K. M. Lakin, "Thin film BAW filters for wide bandwidth and high performance applications," IEEE Microwave Theory and Techniques Symp. Dig., 2004, pp. 923-926.

[2] G. G. Fattinger, J. Kaitila, R. Aigner, and W. Nessler, "Single-tobalanced filters for mobile phones using coupled resonators BAW technology," IEEE Ultrasonic Symp., UFFC Joint 50th Anniversary Conf., Montreal, 2004, pp. 416-419.

[3] T. Uno, "Bulk acoustic wave filters using piezoelectrically coupled resonators," Jpn. J. Appl. Phys., vol. 33, no. 5B, part 1, pp. 30013004, 1994.

[4] R. P. Kampen and R. F. Wolffenbuttel, "Modeling the mechanical behaviour of bulk micromachined silicon accelerometers.," Sens. Actuators A, vol. 64, no. 2, pp. 137-150, 1998.

[5] P. Scheeper, J. O. Gullov, and L. M. Kofoed, "A piezoelectric triaxial accelerometer," J. Micromech. Microeng., vol. 6, no. 1, pp. 131-133, 1996.

[6] H. Kueppers, T. Leuerer, U. Schnakenberg, W. Mokwa, M. Hoffmann, T. Schneller, U. Boettger, and R. Waser, "PZT thin film for piezoelectric microactuator applications," Sens. Actuators A, vol. 97-98, pp. 680-684, Apr. 2002.

[7] K. Prume, P. Muralt, F. Calame, T. Schmitz-Kempen, and S. Tiedke, "Piezoelectric thin film: evaluation of electrical and electromechanical characteristics for MEMS devices," IEEE Trans. Ultrason., Ferroelectr., Freq. Control, vol. 54, no. 1, pp. 8-14, 2007.

[8] M.-A. Dubois and P. Muralt, "Measurement of the effective transverse piezoelectric coefficient $e_{31, f}$ of $\mathrm{AlN}$ and $\mathrm{Pb}\left(\mathrm{Zr}_{x}, \mathrm{Ti}_{1-x}\right) \mathrm{O}_{3}$ thin films," Sens. Actuators A, vol. 77, no. 2, pp. 106-112, 1999.

[9] E. Cattan, T. Haccart, and D. Remiens, " $e_{31}$ piezoelectric constant measurement of lead zirconate titanate thin films," J. Appl. Phys., vol. 86, no. 12, pp. 7017-7023, 1999.

[10] J. Yang, The Mechanics of Piezoelectric Structures. Singapore: World Scientific Publishing, 2006.

[11] R. D. Mindlin, An Introduction to the Mathematical Theory of Vibrations of Elastic Plates. J. Yang, Ed. New Jersey: World Scientific, 2006.

[12] M. N. Niu and E. S. Kim, "Piezoelectric bimorph microphone built on micromachined parylene diaphragm," J. Microelectromech. Syst., vol. 12 , no. 6, pp. 892-898, 2003.

[13] J. M. Whitney, Structural Analysis of Laminated Anisotropic Plates. Lancaster, PA: Technomic, 1987.

[14] P. Muralt, A. Kholkin, M. Kohli, T. Maeder, and N. Setter, "Characterisation of PZT thin films for micromotors," Microelectron. Eng., vol. 29, no. 1-4, pp. 67-70, Dec. 1995.

[15] M. Zhu and P. B. Kirby, "Design study of piezoelectric micro-machined mechanically coupled cantilever filters using a combined finite element and microwave circuit analysis," Sens. Actuators A, vol. 126, pp. 417-424, 2006.

[16] M. Zhu,, P. B. Kirby, and M.Y. Lim, "Lagrange's formulism for modeling of a triaxial microaccelerometer with piezoelectric thinfilm sensing," IEEE Sensors J., vol. 4, no. 4, pp. 455-463, 2004. 


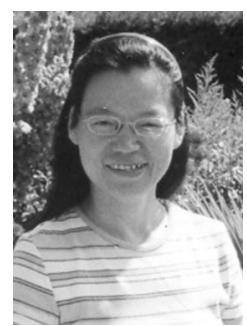

Meiling Zhu received the Ph.D. degree at the Department of Mechanical Engineering, Southeast University, Nanjing, China, in 1995

After the completion of this work, she moved to the Institute of Vibration Engineering Research in Nanjing University of Aeronautics \& Astronautics to investigate piezoelectric motors. In recognition of the value of her work in the area of piezoelectric motors, the Alexander von Humboldt Foundation in Germany awarded her a fellowship at Stuttgart University between 1999 and 2001. Since 2002, she has been at the Microsystems and Nanotechnology Centre at the School of Applied Science of Cranfield University, working in the area of piezoelectric sensing and actuation and energy harvesting. Her expertise is mainly in design, analysis, modeling and simulation, prototyping, and applications of macro/micro-sized piezoelectric devices.

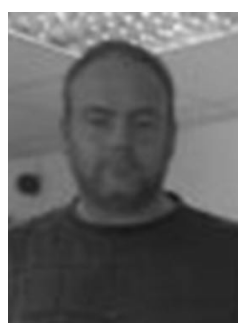

Glenn Leighton was born in Doncaster, England, in 1970. After leaving school he did an apprenticeship as a mechanical engineer and worked in various roles until returning to education. $\mathrm{He}$ received the HND degree in electronics and advanced computing technology from Doncaster College in 2000 and the B.Sc. degree in integrated technology from Hull University in 2001. After completing the M.Sc. degree in microsystems and nanotechnology in 2004, he stayed on at Cranfield University to attain the Ph.D. degree in RF MEMS devices in 2008.

His research skills lie in the use of advanced metrology for piezoelectric materials characterization, electronics, design of MEMS devices, FEA modeling, and fabrication of devices. 